\title{
Analysis of the Interaction of Space Tethers with Catalogued Space Objects
}

\author{
Robert Hoyt, Jason Buller, \\ Tethers Unlimited, Inc., Bothell, WA, 98011 \\ www.tethers.com
}

\begin{abstract}
The potential for collisions or close passes with other space objects presents a significant issue for many space tether applications, representing a potential risk both to the integrity of the tether system and to the safety of other spacecraft. Potential collisions between tethers and other space objects may be possible to avoid if close encounters can be predicted with sufficient precision and advance notice. In order to provide a method for predicting the frequency with which a tether must be maneuvered to avoid collisions, and to provide a resource for accurate close-encounter prediction during tether flight experiments, we have developed a software tool that compares the trajectory of a tether object with that of all of the objects in the NORAD space catalogue. In this paper we describe the models and algorithms used in this tool, and discuss results of test cases conducted to predict the close-encounter frequency of a tether systems ranging from a short nanosatellite-based tether experiment to a hundred-kilometer long MXER tether system.
\end{abstract}

\section{INTRODUCTION}

Space tether systems are of significant interest for a number of space transportation and scientific research applications, including momentum-exchange propulsion for orbital transfer, ${ }^{1,2}$ electrodynamic propulsion for orbit-raising, end-of-mission deorbit, and plane changes, ${ }^{3,4}$ and investigations of the upper atmosphere. ${ }^{5}$ Because many tether system concepts will utilize tether structures with lengths of tens or even hundreds of kilometers, these tethered systems can have higher collision cross-sections than traditional space systems, which typically have dimensions of only a few tens of meters at most. As a result, understanding and monitoring the collision risk for a tethered system will be necessary both in its design and operational phases.

Understanding and minimizing collision risks is of particular importance to the "momentumexchange/electrodynamic-reboost" (MXER) Tether Transport System concept, in which a 80-120 km long rotating tether in Earth orbit would be used to pick payloads up from LEO and toss them into transfer trajectories to GEO, the Moon, and deep space. With such a long tether passing through the (relatively) densely populated regions of low-Earth orbit, the potential for close encounters with existing spacecraft and space debris is non-trivial, and thus in order to ensure that a MXER tether can survive for a number of years and does not cause damage to other space systems, the operational segment of the tether system will be required to continually compare the predicted path of the tether to the predicted paths of the catalogue of existing space objects, and command the tether system to perform maneuvers to avoid any predicted close passes. The frequency with which such maneuvers must be performed will have a significant impact upon the operational costs of such a system.

\section{A. Prior Work}

Several prior efforts have developed methods for calculating the collision probability between a space tether and another space object. Patera used a method in which the positional error of a tether and another spacecraft were represented by a three-dimensional Gaussian probability density to compare the collision probability between a 1-m sphere and a $20-\mathrm{km}$ tether with the collision probability between a $1-\mathrm{m}$ sphere and a $6-\mathrm{m}$ sphere, and found that the $20 \mathrm{~km}$ tether had a collision probability roughly 200-300 times higher than the 6-m sphere. ${ }^{6}$ Cooke et al. calculated the interaction rate between a $20 \mathrm{~km}$ tether and the existing space object catalogue in two different manners, one based on an analytic method developed by Matney, and the other a "brute force" method in which the tether's orbit was propagated and compared to the orbits of the catalogued objects. ${ }^{7}$ In these analyses Cooke found that a $20 \mathrm{~km}$ tether in a low inclination, $400 \mathrm{~km}$ orbit will pass within half a kilometer of another object roughly 9 times per year, whereas a tether in a high inclination, $800 \mathrm{~km}$ orbit may pass within 500 meters of another object almost once per 
day. In these analyses, the tethers were assumed to be in circular orbits, and the tether was assumed to be aligned along the local vertical.

\section{B. Tether Conjunction Analysis}

For future space tether applications, once the tether is delivered into orbit, the cost of operations for the mission will be driven largely by the resources required to monitor and control the tether. For example, if a MXER Tether Transportation System were to experience very frequent close-passes with other space objects, the costs for the manpower, sensing, and computational resources needed to constantly monitor and maneuver the tether to avoid these passes could detract from the potential transportation cost savings offered by the system. In order to determine the resources needed to operate space tether systems, we have developed tools for estimating the frequency of close encounters between space tethers and other catalogued objects. These tools are similar to the "brute force" method employed by Cooke's analysis, but have been extended to allow the analysis of tethers having rotation or other dynamical behavior, as well as to enable conjunction rates to be calculated as a function of minimum distance. In these tools, the orbit and dynamic behavior of the tether is propagated over a specified period of time, and the tether's trajectory is compared to that of other objects in the Space-Track catalogue. ${ }^{8}$ In this paper we will describe the algorithms used to perform these analyses, and present results for analyses of two tether systems currently in development: first, a $1-\mathrm{km}$ long CubeSat-based tether experiment, and the second a $100-\mathrm{km}$ long rotating MXER tether system.

\section{Tether-Satellite ConJunction ANalysis Method}

In order to enable rapid analysis of the frequency with which a tether system will encounter other space objects, we have developed a software tool that propagates the orbits of one or more tether systems and compares its trajectory to a catalogue of satellites. This tool, called "Conjunctionator", has been implemented in a manner suitable for deployment on a grid-computing architecture such as XGrid. ${ }^{9}$ This grid-enabled implementation permits conjunction analyses to be performed against the roughly 10,000 objects catalogued by the U.S. Space Command at speeds many times real time, making it suitable for use as a part of an operations package for space tether missions.

\section{A. Satellite Propagation}

To propagate the orbital dynamics of the satellite catalogue, the Conjunctionator code uses the standard SGP and SDP algorithms used for propagation of NORAD element sets. ${ }^{10}$

\section{B. Tether Propagation}

To propagate the tether, two different models can be used. In the simplest model, the SGP/SDP algorithms are used to propagate the orbit of the center of gravity of the tether system, and positions of the endpoints of the tether are computed using a simple rigid-rod model. For more accurate analyses, or in operational applications, the Conjunctionator code can accept data sets on tether motion and dynamics generated by the TetherSim ${ }^{\mathrm{Tu}} \operatorname{code}{ }^{\prime \prime}$

\section{Conjunction Analysis}

In a tether conjunction analysis, the positions of the catalogue satellites are calculated for the beginning and end of each timestep, and the trajectory of each satellite over the timestep is assumed to be a straight line. Typically, timesteps of a second are chosen to minimize errors due to this assumption. The tether position at the beginning and end of the timestep is then calculated; for very long tethers or tethers exhibiting dynamics, we also determine the positions for several nodes spaced along the tether. Then, for each satellite in the catalogue, the code calculates the distance from the tether's center of gravity to the satellite at each timestep. This distance is then compared to a threshold value, typically chosen to be several times the length of the tether for a long tether system $(\geq 2 \mathrm{~km})$, or, for a shorter tether, ten times the distance the tether will travel over a timestep. If the distance is below this threshold value, the satellite and tether positions are calculated at substep intervals to determine the time and closest approach distance between the satellite and any portion of the tether. Conjuctions with a passing distance below a specified threshold are then recorded for further analysis

\section{Tether-Satellite Conjunction Analysis Results}

We have used the Conjunctionator tool to simulate two candidate tether missions, one a relatively short, 1-km gravity-gradient oriented tether in a polar LEO orbit, and the other a $100-\mathrm{km}$ long rotating momentum exchange tether in a elliptical, equatorial orbit. 


\section{A. MAST Experiment}

The Multi-Application Survivable Tether (MAST) Experiment is a small, low-cost mission that will deploy three CubeSats along a $1-\mathrm{km}$ long survivable tether. ${ }^{12}$ This experiment is planned to fly as a secondary payload in late $2005 /$ early 2006 , in a polar orbit with an initial altitude in the range of $500-600 \mathrm{~km}$. Because the MAST tether and CubeSats will have a very large ratio of drag-area to mass, it will have a relatively short lifetime, in the range of 3-9 months. In order to evaluate the conjunction frequency of this tether with other space objects, we propagated a $1 \mathrm{~km}$ long gravity-gradient oriented tether in a $600 \mathrm{~km}$ orbit for a period of a year. For the satellite catalogue, we utilized a Cheyenne Mountain Operations Center 2-line element set generated in December of 2003, which contained all of the non-classified space objects being tracked by the CMOC at that time, a total of 8,545 objects. In order to evaluate the impact of deploying such a tether from a satellite upon its conjunction frequency, we also propagated a $10-$ meter sized satellite against the same population. The frequency of conjunctions with other catalogue objects is plotted as a function of closest approach distance in Figure 1 for both the MAST tether and the canonical 10-m satellite. In that 1-year period, neither the MAST tether nor the $10-\mathrm{m}$ satellite passed within $1 \mathrm{~km}$ of any other catalogue object. Over a six-month mission lifetime, the tether can be expected to pass within $2 \mathrm{~km}$ of two or three objects. During the 1-year simulation time, the tether passed within $10 \mathrm{~km}$ of 122 objects, while the satellite passed within 10 $\mathrm{km}$ of 116 objects. From these results, we conclude that deploying a $1-\mathrm{km}$ length tether will result in only a $5 \%$ increase in the number of conjunctions of $10 \mathrm{~km}$ or less.

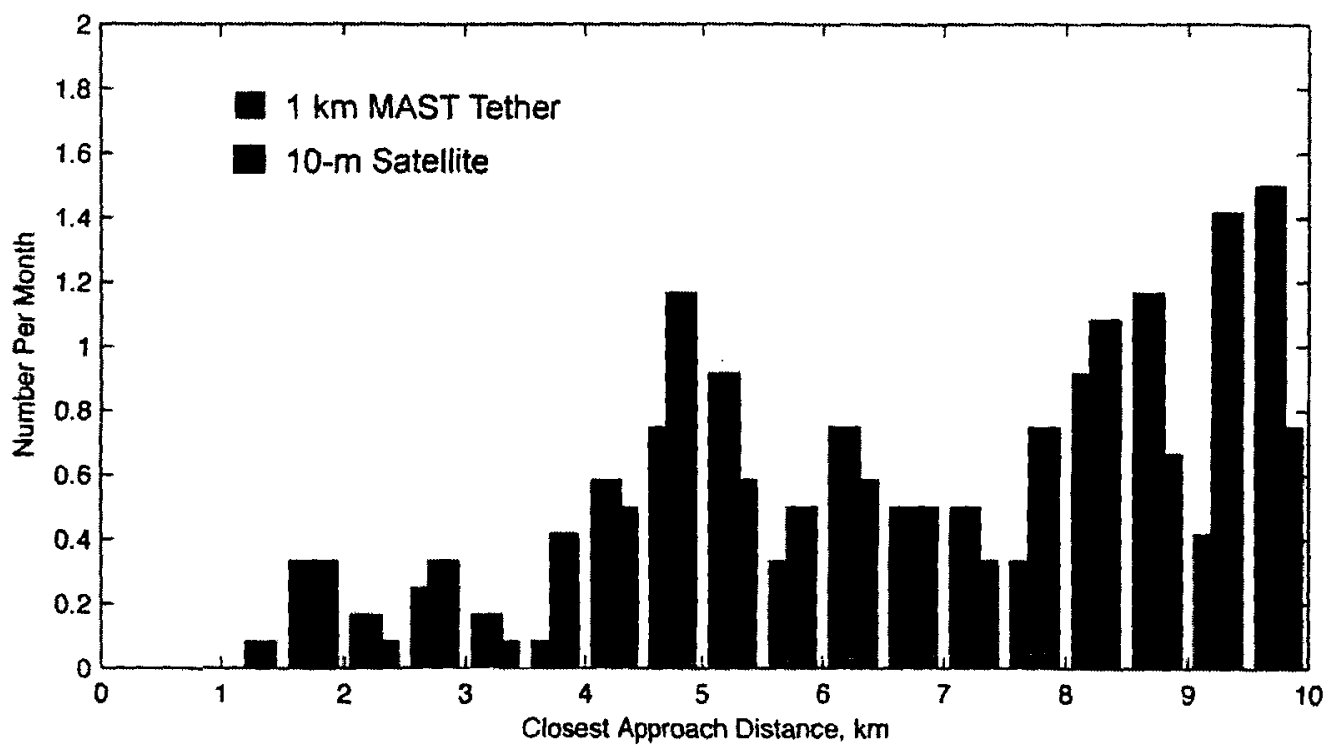

Figure 1. Comparison of the rates at which a 1-km tether and a $10-\mathrm{m}$ satellite in a $600 \mathrm{~km}$ polar orbit will encounter other catalogue objects, as a function of closest approach distance.

\section{B. MXER Tether Boost Facility}

The MXER Tether Boost Facility will be a $100 \mathrm{~km}$ long tether structure that will orbit the Earth in an equatorial, elliptical orbit with a perigee in low-LEO and an apogee of approximately $9,000 \mathrm{~km}$ altitude. Because of its exceptional length, there has naturally been significant concern regarding its potential for collisions with other spacecraft. It is expected that the operations of the MXER tether would include continuous monitoring prediction of the tether's orbit, and if a close pass is predicted, maneuvers such as thrusting or tether reeling would be performed to avoid the other object. If such maneuvers are required only infrequently, the impact upon operations costs will be low, but if such maneuvers are required very frequently, then the costs for such operations could be a significant driver on the operations costs of the system, and planning of each maneuver could become quite complicated as each maneuver must be carefully chosen to avoid causing a conjunction with a different object.

Using the Conjunctionator tool, we simulated a $100-\mathrm{km}$ tether in an elliptical orbit with semimajor axis of 10,560 $\mathrm{km}$ and eccentricity of 0.334 , an inclination of $0^{\circ}$, and a rotation period of 10 minutes, and propagated it over a pe- 
riod of two years against a set of 10,709 two-line elements obtained from the Space-Track website on 17 January 2005.

Figure 2 shows the average number of conjunctions per month plotted as a function of the closest approach distance. These data show that although the MXER tether is quite long, and does pass through the relatively densely populated LEO altitudes, because it is in an equatorial orbit, where there are few LEO satellites, and because it spends only a small fraction of its orbit period within LEO, the frequency of its encounters with other space objects will be relatively low. In fact, a comparison of the conjunction rates for the MXER tether in Figure 2 with the conjunction rates for the 10-m spacecraft in Figure 1 reveals the somewhat surprising result that the MXER tether will have a conjunction rate roughly comparable to a conventional LEO spacecraft.

Nonetheless, because the dynamic and orbital behavior of a tether system is more complicated than that of a conventional satellite, the uncertainty in propagating the tether will be greater than that for a satellite. The threshold for predicted closest approach distance will depend upon how accurately a tether can be propagated by simulation tools, and thus the net impact upon the operations costs for a MXER tether system will depend upon the accuracy of the systems used to monitor, predict, and control the tether system. Fortunately, recent analyses of simulation methods for MXER tethers have indicated that simulation accuracies on the order of meters-per-orbit may be feasible, ${ }^{13}$ so there is strong potential that ultimately the threshold for closest approach distance can be reduced to levels where close-pass avoidance maneuvers will need to be performed only once every several months.

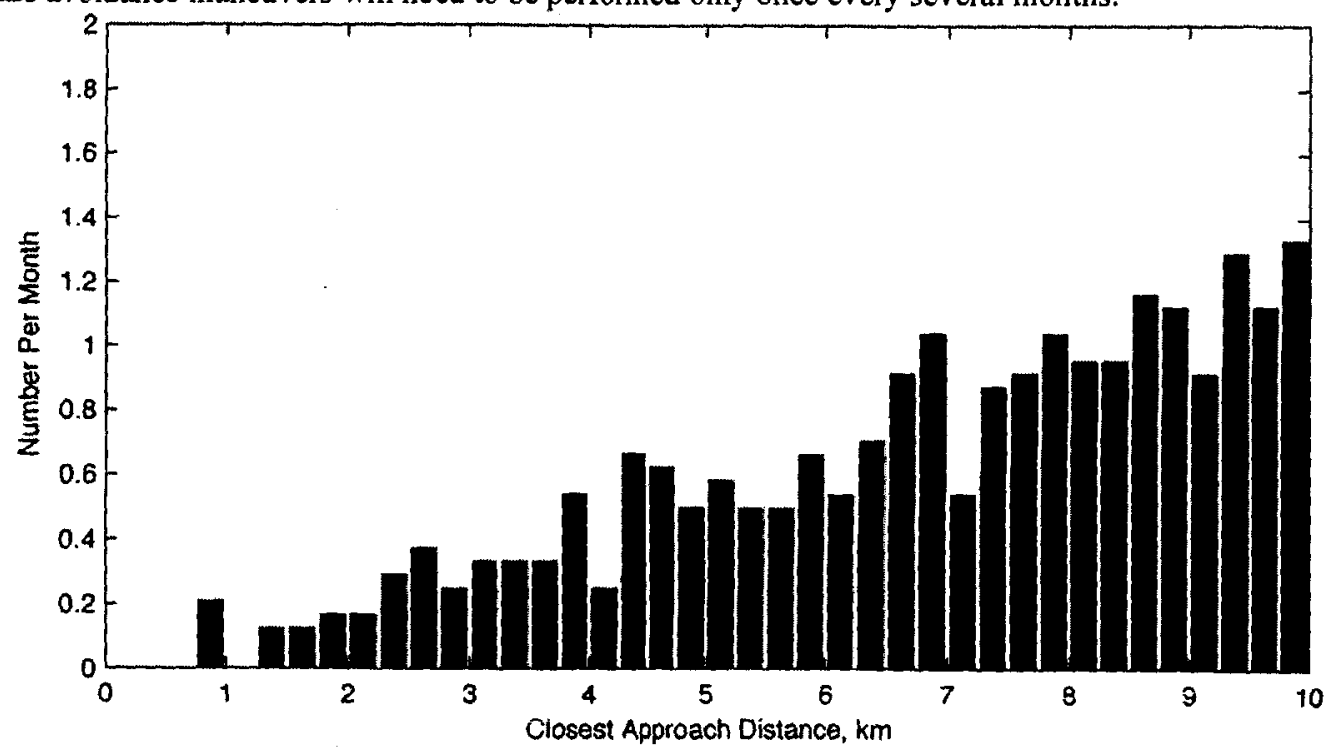

Figure 2. Rate at which the $100 \mathrm{~km} \mathrm{MXER} \mathrm{Tether} \mathrm{will} \mathrm{encounter} \mathrm{other} \mathrm{catalogue} \mathrm{objects,} \mathrm{as} \mathrm{a} \mathrm{function} \mathrm{of}$ the closest approach distance. Data shown represents an average over two years of simulated interaction with the Space-Track catalogue.

\section{CONCLUSIONS}

We have developed a software tool for simulating the orbital motion of tether systems and comparing their trajectories to those of space objects described by the commonly used CMOC two-line element set. This "Conjunctionator" tool has been deployed on a grid-computing platform to enable rapid analysis as a part of flight mission operations. Simulations of a $1-\mathrm{km}$ tether in a polar LEO orbit and a $100 \mathrm{~km}$ tether in an elliptical, equatorial orbit indicate that the rate of conjunctions with other catalogued objects for these two tethers is not greatly larger than that of a conventional satellite in a polar LEO orbit. The results also indicate that developing capabilities for high-precision monitoring and prediction of the orbital and dynamic motions of a tether system can provide a strong payoff in reducing the operations costs for a tether system such as the MXER Tether Transport System. 


\section{ACKNOWLEDGEMENTS}

The work described in this paper was funded in whole by a NASA/MSFC Phase II SBIR contract, number NNM04AA10C, COTR Dr. Joseph Bonometti, through the In-Space Propulsion Technology Program, which is managed by NASA's Science Mission Directorate in Washington, D.C., and implemented by the In-Space Propulsion Technology Office at Marshall Space Flight Center in Huntsville, Ala. The program objective is to develop inspace propulsion technologies that can enable or benefit near and mid-term NASA space science missions by significantly reducing cost, mass or travel times. The authors also wish to acknowledge the assistance of Dr. Robert Racca of the AFSPC Space Analysis Center (ASAC).

\section{REFERENCES}

1. Hoyt, R.P., Slostad, J.T., Frank, S.S., "A Modular Momentum-Exchange/Electrodynamic-Reboost Tether System Architecture," AIAA Paper 2003-5214, 39 'th Joint Propulsion Conference, Huntsville, AL, July 2003.

2. Sorensen, K., 2001, "Conceptual Design and Analysis of an MXER Tether Boost Station," AIAA Paper 20013915.

3. Forward, R.L., Hoyt, R.P., and Uphoff, C.W., "The Terminator Tether": A Spacecraft Deorbit Device", $J$. Spacecraft and Rockets, 37(2) March-April 2000.

4. Pearson, J., et al., "Orbital Maneuvering with Spinning Electrodynamic Tethers," AlAA Paper 2004-5715, 2nd International Energy Conversion Engineering Conference, 16 - 19 August 2004, Providence, Rhode Island .

5. Pradeep, S., Kumar, K., "Extension of tethered satellites in the atmosphere," Acta Astronautica, 52, 2003, pp. 110.

6. Patera, R.L., "Method for Calculating Collision Probability Between a Satellite and a Space Tether," Journal of Guidance, Control, and Dynamics, 25(5), p 940-945, 2002.

7. Cooke, W.J., et al., "Tether Survivability and Collision Avoidance: Is LEO the Right Place for Tethered Systems?", STAIF-2001, Feb 2001.

8. http://www.space-track.org/

9. XGrid Guide, Apple Computer, 17 March 2004.

10. Hoots, Felix R. and Roehrich, Ronald L., "Models for Propagation of NORAD Element sets," Spacetrack Report No. 3, compiled by T.S. Kelso, 31 Dec., 1988.

11. TetherSim User's Guide, Tethers Unlimited, Inc., June 2004.

12. Hoyt, R.P., Slostad, J.T., Twiggs, R., "The Multi-Application Survivabie Tether (MAST) Experiment", AIAA Paper 2003-5219, 39 $9^{\text {ih }}$ Joint Propulsion Conference, Huntsville, AL, July 2003.

13. Levin, E.M., "MXER Simulation Study," STAR, Inc. report to NASA/MSFC, 10 Jan 2005. 